\title{
Aufbruch nach Pandora
}

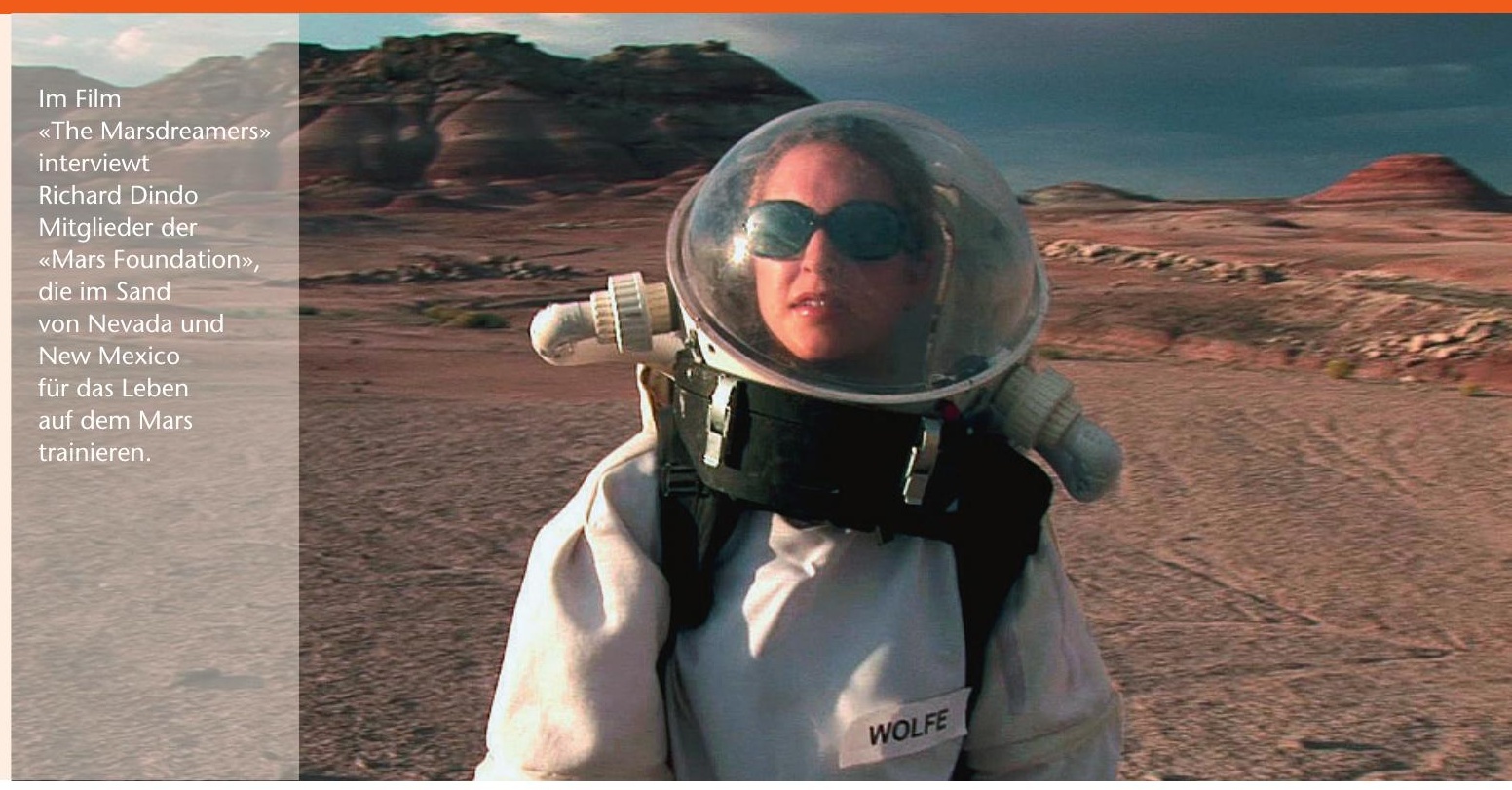

Erhard Taverna
Auch die 45. Solothurner Filmtage boten das gewohnte Bild sonnig-kalter Januartage, bekannter Einführungsrituale und besorgter Forumsgespräche vor einer ruhig dahinflutenden Aare. Es gab über 300 Filme zu sehen, darunter wie immer einige medizinische, weniger als auch schon, nebst vielen Familiendramen, einige berührende Porträts aussergewöhnlicher Menschen und eine spannende «Rencontre» mit Niki Reiser, dem vielfach preisgekrönten Schweizer Musiker und Filmkomponist.

Die gescheiterte Klimakonferenz in Kopenhagen und das UNO-Jahr der Biodiversität hatte auch hier für ein filmisches Vor- und Nachbeben gesorgt. Doch anders als im Katastrophenkracher «2012» von Roland Emmerich, den man nachträglich als eine Art globales Super-Haiti bezeichnen könnte, sind es die leiseren und gedankenvolleren Töne, die in Solothurn nachdenklich stimmten. Wo Emmerich sich als kontinentaler Abbruchspezialist profiliert, der am Ende drei Archen nach Afrika steuert, zeigt die 1966 in Weinfelden geborene Regisseurin Mirjam von Arx in «Seed Warriors» das Projekt einer Saatgut-Arche in der Arktis. In drei riesigen Eiskammern Norwegens wollen Wissenschaftler das Saatgut der rund 1400 von der FAO registrierten Genbanken lagern. Eine Backup-Alternative für die wichtigsten samenproduzierenden Gemüseund Früchtepflanzen zu den bereits heute im tiefgekühlten Tresor ruhenden Samen. Einige werden Tausende von Jahren überstehen, andere nur wenige Jahrzehnte. Das vom norwegischen Staat unterstützte «Svalbard Global Seed Vault Project» versteht sich darum als Teil eines Netzwerkes, das ununterbrochen für den überlebenswichtigen Nachschub an keimfähigen Pflanzen sorgt, wenn Temperaturanstieg und Bevölkerungswachstum das Überleben der Menschen bedrohen.

Als Vorfilm lief wie ein prophetischer Albtraum die Videoarbeit der in Zürich arbeitenden Amerikanerin Elodie Pong. Im bereits an den Winterthurer-Kurzfilmtagen prämierten Streifen «After The Empire» diskutieren vor industriellen Ruinenkulissen längst verstorbene oder fiktive Gestalten aus Politik und Popkultur. Monroe, Marx, Elvis und Batman berichten wie Zeugen einer untergegangenen Welt von ihren Idealen, Utopien, Sehnsüchten und Hoffnungen.

Mitglieder der «Mars Foundation» planen wie die Pilgerväter der «Mayflower» einen Exodus zum roten Planeten. Der bekannte Dokumeister Richard Dindo hat sie in den USA getroffen und vor laufender Kamera befragt. Eine Geologin, eine Höhlenforscherin und Astrobiologin, ein NASA-Spezialist, ein Informatiker, ein Architekt und viele andere träumen und trainieren im Sand von Nevada und New Mexico das neue Leben in staubsicheren Raumanzügen und strahlengeschützten Behausungen. Sie sind begeistert vom Kolonistendasein künftiger «new frontier Generationen», die in ihren Gewächshäusern eine utopische Gesellschaft aufbauen. «The Marsdreamers» erzählt ironisch, aber respektvoll vom Enthusiasmus widersprüchlicher, sympathischer Menschen, die alle ihre Träume auf einen unwirtlichen Ersatzplaneten projizieren, während zwei befragte Indianer aus leidvoller Erfahrung zum respektvollen Umgang mit dem Unbekannten ermahnen. Richard Dindo vermischt in grandiosen Bildern terrestrische und marsianische Felswüsten zum Gesamtbild einer bedrohten Erde, die Männer und Frauen lieber schon heute als morgen verlassen möchten.

Überlebende, wie im Film «Cargo» von Ivan Engler, haben diese nach dem Öko-Kollaps bereits verlassen und vegetieren in überfüllten Raumstationen. Alles ist hier düster und heruntergekommen, tiefgefroren im Kälteschlaf oder auf einsamer Nachtschicht, wie die junge Ärztin Laura auf dem gespenstisch leeren Raumfrachter Kassandra, unterwegs zum Lichtjahre entfernten, paradiesischen Planeten Rhea. Ein bildstarker Ostschweizer-Beitrag mit berühmten Vorbildern, aber sehr viel weniger Geld. Ebenso viele Lichtjahre entfernt vom bisher teuersten aller Hollywoodfilme «Avatar» von James Cameron, der seit Dezember 2009 in allen grossen Kinos der Welt läuft. Fünf Millionen Kinobesucher im ersten Monat allein in Deutschland, diese astronomische Zahl wird kein Schweizer Spielfilm je 
erreichen. Doch der Vergleich ist natürlich nicht fair. Die Geschichte des technisch brillanten Grossspektakels mit den real gedrehten und computeranimierten Szenen liegt auf der gleichen Linie wie die erwähnten Beispiele. Die Rohstoffe der Erde sind erschöpft, die Menschen beginnen das Weltall auszubeuten. Pandora, ein erdähnlicher Mond eines fiktiven Planeten Polyphemus im Sternsystem Alpha Centauri, wird nach einer gescheiterten Missionierung zum Schauplatz eines militärischen Eroberungsversuches. Die Fieslinge kommen von der Erde, die Guten reden wie der Häuptling Papalagi. Nur sind die Indianer jetzt Blauhäute, dafür am Ende erfolgreich. Ein Ethno-Esoterik-Western, grosses Abenteuerkino mit 3-D-Spezialeffekten. Konservativen US-Politikern missfällt das Drehbuch, und Chinas Führung mag es auch nicht. Das macht alle diese Filme schon fast wieder glaubwürdig und damit leider auch die mehr oder weniger pessimistische
Sicht auf unsere Fähigkeiten, im Einklang mit der Vielfalt des eigenen Planeten zu leben. Führer mögen es nicht, wenn Untertanen ihre Weisheiten bezweifeln. Die berechtigte Sorge über deren Unvermögen und das damit verbundene eigene Schicksal fand auch an den Solothurner Filmtagen einen intelligenten und vielseitigen Ausdruck.

\section{Besprochene Filme:}

- «Seed Warriors», Regie Mirjam von Arx Drehbuch Katharina von Flotow, 86 Minuten.

- «After the Empire», Regie und Drehbuch Elodie Pong, 15 Minuten.

- «The Marsdreamers», Regie Richard Dindo, 83 Minuten.

- «Cargo», Regie Ivan Engler, 122 Minuten.

SWISS MEDICAL WEEKLY

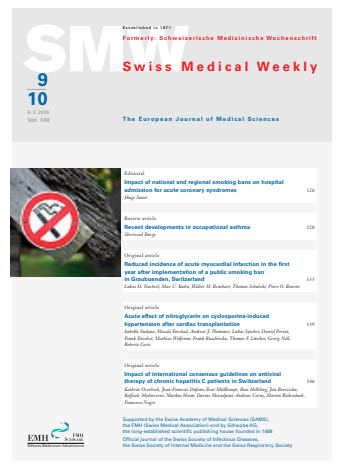

Das «Swiss Medical Weekly» ist eine international beachtete, peer reviewte Forschungszeitschrift auf Open-Access-Basis. Mit Ihrem Abonnement der gedruckten Ausgabe unterstützen Sie diese insbesondere für den akademischen Nachwuchs in der Schweiz wichtige wissenschaftliche Plattform:

Jahresabonnement (25 Ausgaben):

Fr. 150.- exkl. Versand

Bestellung per E-Mail an auslieferung@ emh.ch, im Internet unter www.smw.ch oder telefonisch unter 0614678575.

\section{Ausgabe 9/10 erscheint am 6. März 2010:}

\section{Recent developments in occupational asthma} Sherwood Burge

Occupational exposures now account for $20 \%$ of adult onset asthma. Overall incidence has not declined, but recognition of the problem and substitutions has resulted in dramatic reductions in some causes of occupational asthma, particularly latex and glutaraldehyde in healthcare workers.

\section{Reduced incidence of acute myocardial} infarction in the first year after implementation of a public smoking ban in Graubuenden, Switzerland

Lukas D. Trachsel, Max U. Kuhn, Walter H. Reinhart, Thomas Schulzki, Piero O. Bonetti

Similar to other countries in Europe and various regions of the USA and Canada, implementation of a public smoking ban was followed by a significant early decline in the incidence of AMI in the Canton of Graubuenden, Switzerland.
Acute effect of nitroglycerin on cyclosporine-induced hypertension after cardiac transplantation Isabella Sudano, Mazda Farshad Tabrizi, Andreas J. Flammer, Lukas Spieker, Daniel Periat, Frank Enseleit, Mathias Wolfrum, Frank Ruschitzka, Thomas F. Luscher, Georg Noll, Roberto Corti

After application of nitroglycerin, sBP is reduced immediately in HTX with uncontrolled cyclosporineinduced hypertension. Further studies are needed to evaluate the long-term effect of nitrates in these patients.

Impact of international consensus guidelines on antiviral therapy of chronic hepatitis C patients in Switzerland

Kathrin Overbeck, Jean-Francois Dufour, Beat Müllhaupt, Beat Helbling, Jan Borovicka, et al., on behalf of the Swiss Hepatitis C Cohort Study Group

Consistent with latest international consensus guidelines, patients enrolled in the Swiss Hepatitis C Cohort with a history of substance abuse were not withheld antiviral treatment. A multidisciplinary approach is warranted to provide antiviral treatment to patients suffering from depression.

EMH Schweizerischer Ärzteverlag Editores Medicorum Helveticorum 